\title{
Coverage with $k$-Transmitters in the Presence of Obstacles
}

\author{
Brad Ballinger ${ }^{1}$, Nadia Benbernou ${ }^{2}$, Prosenjit Bose ${ }^{3}$, Mirela Damian*4, \\ Erik D. Demaine ${ }^{5}$, Vida Dujmović ${ }^{6}$, Robin Flatland ${ }^{7}$, Ferran Hurtado $^{\dagger 8}$, \\ John Iacono $^{9}$, Anna Lubiw ${ }^{10}$, Pat Morin ${ }^{11}$, Vera Sacristán ${ }^{\dagger 12}$, \\ Diane Souvaine ${ }^{13}$, and Ryuhei Uehara ${ }^{14}$ \\ 1 Humboldt State University, Arcata, USA, brad.ballinger@humboldt.edu \\ 2 Massachusetts Institute of Technology, Cambridge, USA, nbenbern@mit.edu \\ 3 Carleton University, Ottawa, Canada, jit@scs.carleton.ca \\ 4 Villanova University, Villanova, USA, mirela.damian@villanova.edu \\ 5 Massachusetts Institute of Technology, Cambridge, USA, edemaine@mit.edu \\ ${ }^{6}$ Carleton University, Ottawa, Canada, vida@cs.mcgill.ca \\ 7 Siena College, Loudonville, USA, flatland@siena.edu \\ 8 Universitat Politècnica de Catalunya, Barcelona, Spain, Ferran.Hurtado@upc.edu \\ 9 Polytechnic Institute of New York University, New York, USA, jiacono@poly.edu \\ 10 University of Waterloo, Waterloo, Canada alubiw@uwaterloo.ca \\ 11 Carleton University, Ottawa, Canada, morin@scs.carleton.ca \\ 12 Universitat Politècnica de Catalunya, Barcelona, Spain, vera.sacristan@upc.edu \\ 13 Tufts University, Medford, USA dls@cs.tufts.edu \\ 14 Japan Advanced Institute of Science and Technology, Ishikawa, Japan \\ uehara@jaist.ac.jp
}

\begin{abstract}
For a fixed integer $k \geq 0$, a $k$-transmitter is an omnidirectional wireless transmitter with an infinite broadcast range that is able to penetrate up to $k$ "walls", represented as line segments in the plane. We develop lower and upper bounds for the number of $k$-transmitters that are necessary and sufficient to cover a given collection of line segments, polygonal chains and polygons.
\end{abstract}

\section{Introduction}

Illumination and guarding problems generalize the well-known art gallery problem in computational geometry $[15,16]$. The task is to determine a minimum number of guards that are sufficient to guard, or "illuminate" a given region under specific constraints. The region under surveillance may be a polygon, or may be the entire plane with polygonal or line segment obstacles. The placement of guards may be restricted to vertices (vertex guards) or edges (edge guards) of the input polygon(s), or may be unrestricted (point guards). The guards may be omnidirectional, illuminating all directions equally, or may be represented as floodlights, illuminating a certain angle in a certain direction.

* Supported by NSF grant CCF-0728909.

† Partially supported by projects MTM2009-07242 and Gen. Cat. DGR 2009SGR1040. 
Inspired by advancements in wireless technologies and the need to offer wireless services to clients, Fabila-Monroy et al. [10] and Aichholzer et al. [2] introduce a new variant of the illumination problem, called modem illumination. In this problem, a guard is modeled as an omnidirectional wireless modem with an infinite broadcast range and the power to penetrate up to $k$ "walls" to reach a client, for some fixed integer $k>0$. Geometrically, walls are most often represented as line segments in the plane. In this paper, we refer to such a guard as a $k$-transmitter, and we speak of covering (rather than illuminating or guarding). We address the general problem introduced in $[10,2]$, reformulated as follows:

k-Transmitter Problem: Given a set of obstacles in the plane, a target region, and a fixed integer $k>0$, how many $k$-transmitters are necessary and sufficient to cover that region?

We consider instances of the $k$-transmitter problem in which the obstacles are line segments or simple polygons, and the target region is a collection of line segments, or a polygonal region, or the entire plane. In the case of plane coverage, we assume that transmitters may be embedded in the wall, and therefore can reach both sides of the wall at no cost. In the case of polygonal region coverage, we favor the placements of transmitters inside the region itself; therefore, when we talk about a vertex transmitter, the implicit assumption is that the transmitter is placed just inside the polygonal region, and so must penetrate one wall to reach the exterior.

\subsection{Previous Results}

For a comprehensive survey on the art gallery problem and its variants, we refer the reader to $[15,16]$. Also see $[9,7,4]$ for results on the wireless localization problem, which asks for a set of 0 -transmitters that need not only cover a given region, but also enable mobile communication devices to prove that they are inside or outside the given region. In this section, we focus on summarizing existing results on the $k$-transmitter problem and a few related issues.

For $k=0$, the $k$-transmitter problem for simple polygons is settled by the Art Gallery Theorem [5], which states that $\left\lfloor\frac{n}{3}\right\rfloor$ guards are sufficient and sometimes necessary to guard a polygonal region with $n$ vertices. Finding the minimum number of 0 -transmitters that can guard a given polygon is NP-hard $[14,15]$. For $k>0$, Aichholzer et al. [10,2] study the $k$-transmitter problem in which the target region is represented as a monotone polygon or a monotone orthogonal polygon with $n$ vertices. They show that $\frac{n}{2 k} k$-transmitters are sufficient, and $\left\lceil\frac{n}{2 k+4}\right\rceil k$-transmitters are sometimes necessary ${ }^{15}$ to cover a monotone polygon. They also show that $\left\lceil\frac{n}{2 k+4}\right\rceil k$-transmitters are sufficient and necessary to cover any monotone orthogonal polygon. The authors also study simple polygons, orthogonal polygons and arrangements of lines in the context of very powerful transmitters, i.e, $k$-transmitters where $k$ may grow as a function of $n$. For example, they show that any simple polygon with $n$ vertices can always be covered

\footnotetext{
$\overline{15}$ The bound $\lceil n /(2 k+2)\rceil$ stated in Theorem 7 from [2] is a typo.
} 
with one transmitter of power $\left\lceil\frac{2 n+1}{3}\right\rceil$, and this bound is tight up to an additive constant. In the case of orthogonal polygons, one $\left\lceil\frac{n}{3}\right\rceil$-transmitter is sufficient to cover the entire polygon. The problem of covering the plane with a single $k$-transmitter has been also considered in [12], where it is proved that there exist collections of $n$ pairwise disjoint equal-length segments in the Euclidean plane such that, from any point, there is a ray that meets at least $2 n / 3$ of them (roughly). While the focus in $[10,2,12]$ is on finding a small number of high power transmitters, our focus in this paper is primarily on lower power transmitters.

The concept of visibility through $k$ segments has also appeared in other works. Dean et al. $[8,13,11]$ study vertical bar $k$-visibility, where $k$-visibility goes through $k$ segments. Aichholzer et al. [1] introduce and study the notion of $k$-convexity, where a diagonal may cross the boundary at most $2(k-1)$ times.

\subsection{Our Results}

We consider several instances of the $k$-transmitter problem. If obstacles are disjoint orthogonal segments and the target region is the entire plane, we show that $\left\lceil\frac{5 n+6}{12}\right\rceil 1$-transmitters are always sufficient and $\left\lceil\frac{n+1}{4}\right\rceil$ are sometimes necessary to cover the target region. If the target region is the plane and the obstacles are lines and line segments that form a guillotine subdivision (defined in $\S 2.2$ ), then $\frac{n+1}{2} 1$-transmitters suffice to cover the target region. We next consider the case where the obstacles consist of a set of nested convex polygons. If the target region is the boundaries of these polygons, then $\left\lfloor\frac{n}{7}\right\rfloor+32$-transmitters are always sufficient to cover it. On the other hand, if the target region is the entire plane, then $\left\lfloor\frac{n}{6}\right\rfloor+3$ 2-transmitters suffice to cover it, and $\left\lfloor\frac{n}{8}\right\rfloor+1$ 2-transmitters are sometimes necessary. All these results (detailed in $\S 2$ ) use point transmitters, with the implicit assumption that transmitters on a boundary segment are embedded in the segment and can reach either side of the segment at no cost.

In Section 3 we move on to the case where the target region is the interior of a simple polygon. In this case, we restrict the placement of vertex and edge transmitters to the interior of the polygon. We show that $\frac{n}{6}$ 2-transmitters are sometimes necessary to cover the interior of a simple polygon. In Section 3.2 we introduce a class of spiral polygons, which we refer to spirangles, and show that $\left\lfloor\frac{n}{8}\right\rfloor$ 2-transmitters are sufficient, and sometimes necessary, to cover the interior of a spirangle polygon. In the case of arbitrary spiral polygons, we derive an upper bound of $\left\lfloor\frac{n}{4}\right\rfloor$ 2-transmitters, matching the upper bound for monotone polygons from $[2]$.

\section{Coverage of Plane with Obstacles}

We begin with the problem of covering the entire plane with transmitters, in the presence of obstacles that are orthogonal segments (§2.1), a guillotine subdivision $(\S 2.2)$, or a set of nested convex polygons $(\S 2.3)$. There is no restriction on the placement of transmitters (on or off a segment). In the case of a transmitter located on a segment itself, the assumption is that the segment does not act as 
on obstacle for that transmitter, in other words, that the transmitter has the power of a $k$-transmitter on both sides of the segment.

\subsection{Orthogonal Line Segments}

In this section the set of obstacles is a set of $n$ disjoint orthogonal line segments and the target region is the whole plane. Czyzowicz et al. [6] proved that $\lceil(n+1) / 2\rceil 0$-transmitters always suffice and are sometimes necessary to cover the plane in the presence of $n$ disjoint orthogonal line segments. We generalize this to $k$-transmitters. Our main ideas are captured by the case of 1 -transmitters, so we begin there:

Theorem 1. In order to cover the plane in the presence of $n$ disjoint orthogonal line segments, $\lceil(5 n+6) / 12\rceil 1$-transmitters are always sufficient and $\lceil(n+1) / 4\rceil$ are sometimes necessary.

Proof. The lower bound is established by $n$ parallel lines - a single 1-transmitter can cover only 4 of the $n+1$ regions.

For the upper bound, the main idea is to remove from the set of segments, $S$, a set of segments that are independent in the sense that no covering ray goes through two of them consecutively. We then take a set of conventional transmitters for the remaining segments. By upgrading these transmitters to 1 -transmitters we cover the whole plane with respect to the original segments $S$.

We now fill in this idea. We will assume without loss of generality that the segments have been extended (remaining interior-disjoint) so that each end of each segment either extends to infinity, or lies on another segment: if a set of $k$-transmitters covers the plane with respect to the extended segments then it covers the plane with respect to the original segments. With this assumption the segments partition the plane into $n+1$ rectangular faces.

The visibility graph $G(S)$ has a vertex for each segment of $S$ and an edge st if segments $s$ and $t$ are weakly visible, i.e. there is a point $p$ interior to $s$ and a point $q$ interior to $t$ such that the line segment $p q$ does not cross any segment in $S$. Equivalently, for the case of extended segments, $s$ and $t$ are weakly visible if some face is incident to both of them.

Lemma 1. If $I$ is an independent set in $G(S)$ and $T$ is a set of 0-transmitters that covers the whole plane with respect to $S-I$, then $T$ is a set of 1-transmitters that covers the whole plane with respect to $S$.

Proof. Suppose that a 0 -transmitter at point $p$ covers point $q$ with respect to $S-I$. Then the line segment from $p$ to $q$ does not cross any segment of $S-I$. It cannot cross two or more segments of $I$ otherwise two such consecutive segments would be visible (and not independent). Thus a 1 -transmitter at $p$ covers $q$ with respect to $S$.

To obtain a large independent set in $G(S)$ we will color $G(S)$ and take the largest color class. If the faces formed by $S$ were all triangles then $G(S)$ would 
be planar and thus 4-colorable. Instead, we have rectangular faces, so $G(S)$ is 1-planar and can be colored with 6 colors. A graph is 1-planar if it can be drawn in the plane, with points for vertices and curves for edges, in such a way that each edge crosses at most one other edge. Ringel conjectured in 1965 that 1planar graphs are 6-colorable. This was proved in 1984 by Borodin, who gave a shorter proof in 1995 [3].
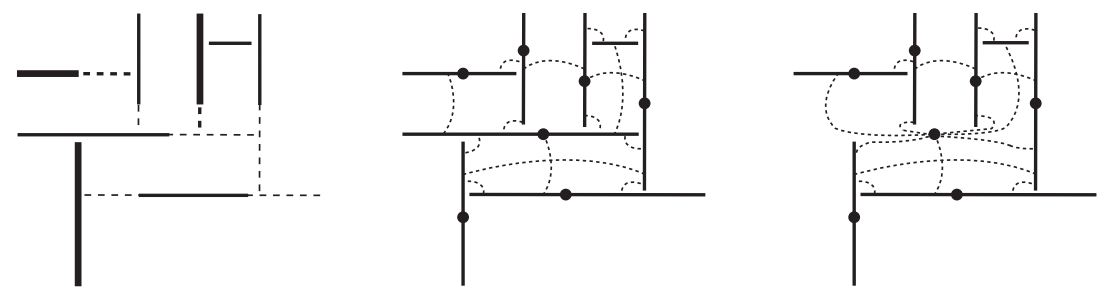

Fig. 1. (left) A set $S$ of disjoint orthogonal segments and their extensions (dashed) with an independent set shown in bold; (middle) $G(S)$ with vertices drawn as segments and edges as dashed curves so 1-planarity is clear; (right) contracting a segment to a point to get a conventional drawing of the graph.

Lemma 2. If $S$ is a set of extended orthogonal segments then $G(S)$ is 1-planar.

Proof. The idea is the same as that used to show that the visibility graph of horizontal line segments is planar. If $G(S)$ is drawn in the natural way, with every vertex represented by its original segment, and every edge drawn as a straight line segment crossing a face, then it is clear that each edge crosses at most one other edge. See Figure 1. We can contract each segment to a point while maintaining this. Note that we end up with a multi-graph in case two segments are incident to more than one face.

We now wrap up the proof of Theorem 1. Since $G(S)$ is 1-planar it has a 6 -coloring by Borodin's result. The largest color class has at least $n / 6$ vertices and forms an independent set $I$. The set $S-I$ has at most $5 n / 6$ segments, so by the result of Czyzowicz et al. [6], it has a set of 0 -transmitters of cardinality at most $\left\lceil\left(\frac{5 n}{6}+1\right) / 2\right\rceil=\lceil(5 n+6) / 12\rceil$ that covers the entire plane. By Lemma 1 , placing 1 -transmitters at those points covers the entire plane with respect to $S$.

We note that the above proof relies on a 6-coloring of $G(S)$. An example that requires 5 colors is shown in Figure 2(a).

Theorem 2. In order to cover the plane in the presence of $n$ disjoint orthogonal line segments, $\left\lceil\frac{1}{2}\left((5 / 6)^{\log (k+1)} n+1\right)\right\rceil k$-transmitters are always sufficient and $\lceil(n+1) / 2(k+1)\rceil$ are sometimes necessary.

Proof. As for $k=1$, the lower bound is realized by parallel segments. One $k$ transmitter can only cover $2(k+1)$ of the $n+1$ regions. 


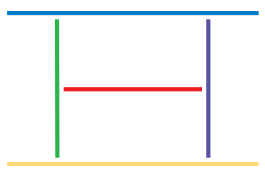

(a)

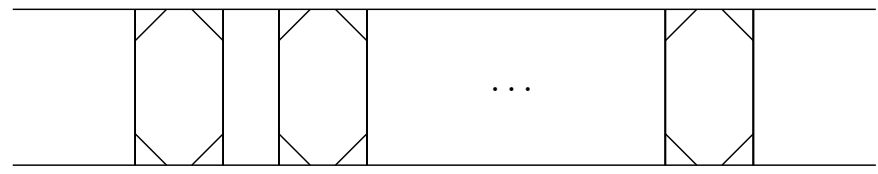

(b)

Fig. 2. (a) An arrangement of five segments whose visibility graph is complete and thus requires 5 colors. (b) A guillotine subdivision with $n=6 k+2$ segments that requires $4 k 0$-transmitters. Each of the $4 k$ triangular faces must have a 0 -transmitter on its boundary and no two triangular faces share a boundary.

For the upper bound, we build on the proof technique for $k=1$. We repeatedly remove independent sets, extending the remaining segments after each removal.

For a set of segments $S$, let $X(S)$ be a set of segments formed by extending those of $S$ until they touch. It will not matter that $X(S)$ is not unique. Let $R_{0}$ be $S$ and for $i=1,2, \ldots$ let $S_{i}$ be a maximal independent set in the visibility graph of $X\left(R_{i-1}\right)$ and let $R_{i}=S-\left(\cup_{j=1}^{i} S_{j}\right)$. Then $R_{i}$ has cardinality at most $(5 / 6)^{i} n$.

Lemma 3. If $T$ is a set of 0-transmitters that covers the whole plane with respect to $R_{i}$, then $T$ is a set of $\left(2^{i}-1\right)$-transmitters that covers the whole plane with respect to $S=R_{0}$.

Proof. We prove by induction on $j=0, \ldots, i$ that $T$ is a set of $\left(2^{j}-1\right)$ transmitters that covers the whole plane with respect to $R_{i-j}$. Suppose this holds for $j-1$. Suppose a $\left(2^{j-1}-1\right)$-transmitter at point $p$ sees point $q$ in $R_{i-j+1}$. Then the line segment $p q$ crosses at most $2^{j-1}-1$ segments of $R_{i-j+1}$, and thus $2^{j-1}$ faces. Consider putting back the segments of $S_{i-j+1}$ to obtain $R_{i-j}$. The segments of $S_{i-j+1}$ are independent in $R_{i-j}$. Therefore the line segment $p q$ can cross at most one segment of $S_{i-j+1}$ in each face. The total number of segments of $R_{i-j}$ crossed by $p q$ is thus $2^{j-1}-1+2^{j-1}=2^{j}-1$. In other words, a $\left(2^{j}-1\right)$-transmitter at $p$ in $R_{i-j}$ covers the same area as the original $\left(2^{j-1}-1\right)$-transmitter at $p$ in $R_{i-j+1}$.

We use this lemma to complete the proof of the theorem. Since we have the power of $k$-transmitters, we can continue removing independent sets until $R_{i}$, where $k=2^{i}-1$, i.e. $i=\log (k+1)$. Then $R_{i}$ has size $(5 / 6)^{\log (k+1)} n$, and the number of 0 -transmitters needed to cover the plane with respect to $R_{i}$ is $\left\lceil\frac{1}{2}\left((5 / 6)^{\log (k+1)} n+1\right)\right\rceil$. Applying the lemma, this is the number of $k$-transmitters we need to cover the plane with respect to $S$.

\subsection{Guillotine Subdivisions}

A guillotine subdivision $S$ is obtained by inserting a sequence $s_{1}, \ldots, s_{n}$ of line segments (possibly rays or lines), such that each inserted segment $s_{i}$ splits a face 
of the current subdivision $S_{i-1}$ into two new faces yielding a new subdivision $S_{i}$. We start with one unbounded face $S_{0}$, which is the entire plane.

As the example in Figure 2(b) shows, a guillotine subdivision with $n$ segments can require $2(n-2) / 30$-transmitters. In this section, we show that no guillotine subdivision requires more than $(n+1) / 21$-transmitters. We begin with a lemma:

Lemma 4. Let $F$ be a face in a guillotine subdivision $S$. If there are 1-transmitters on every face that shares an edge with $F$ then these 1-transmitters see all of $F$.

Proof. Consider the segment $s_{i}$ whose insertion created the face $F$. Before the insertion of $s_{i}$, the subdivision $S_{i-1}$ contained a convex face that was split by $s_{i}$ into two faces $F$ and $F^{\prime}$ (Figure 3(a)). No further segments were inserted into $F$, but $F^{\prime}$ may have been further subdivided, so that there are now several faces $F_{1}^{\prime}, \ldots, F_{k}^{\prime}$, with $F_{j}^{\prime} \subseteq F^{\prime}$ and $F_{j}^{\prime}$ incident on $s_{i}$ for all $j \in\{1, \ldots, k\}$ (Figure 3(b)).

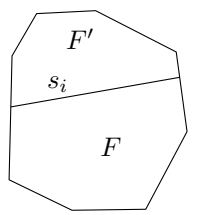

(a)

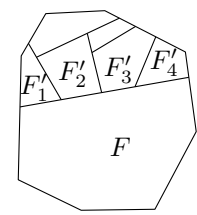

(b)

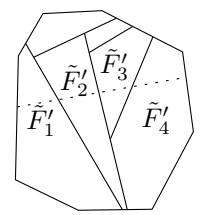

(c)

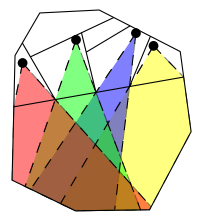

(d)

Fig. 3. The proof of Lemma 4.

We claim that the 1 -transmitters in $F_{1}^{\prime}, \ldots, F_{k}^{\prime}$ guard the interior of $F$. To see this, imagine removing $s_{i}$ from the subdivision and instead, constructing a guillotine subdivision $\tilde{S}$ from the sequence $s_{1}, \ldots, s_{i-1}, s_{i+1}, \ldots, s_{n}$ (Figure 3(c)). In this case, each face $F_{j}^{\prime}$ in $S$ becomes a larger face $\tilde{F}_{j}^{\prime}$ in $\tilde{S}$ and together $\bigcup_{j=1}^{k} \tilde{F}_{j}^{\prime} \supseteq F$. Finally, we observe that each 1 -transmitter in $S$ in face $F_{j}^{\prime}$ guards at least $\tilde{F}_{j}^{\prime}$, so together, the 1 -transmitters in $F_{1}^{\prime}, \ldots, F_{k}^{\prime}$ guard all of $F$ (Figure $3(\mathrm{~d}))$.

Theorem 3. Any guillotine subdivision can be guarded with at most $(n+1) / 2$ 1-transmitters.

Proof. Consider the dual graph $T$ of the subdivision. $T$ is a triangulation with $n+1$ vertices. Let $M$ be any maximal matching in $T$. Consider the unmatched vertices of $T$. Each such vertex is adjacent only to matched vertices (otherwise $M$ would not be maximal). Let $G$ be the set of 1 -transmitters obtained by placing a single 1-transmitter on the primal edge associated with each edge $e \in M$. Then $|G|=|M| \leq(n+1) / 2$. For every face $F$ of $S, F$ either contains a 1 -transmitter in $G$, or all faces that share an edge with $F$ contain a 1 -transmitter in $G$. In the former case, $F$ is obviously guarded. In the latter case, Lemma 4 ensures that $F$ is guarded. Therefore, $G$ is a set of 1 -transmitters that guards all faces of $F$ and has size at most $(n+1) / 2$. 


\subsection{Nested Convex Polygons}

The problems analyzed in this section are essentially two:

1. How many 2-transmitters are always sufficient (and sometimes necessary) to cover the edges of a set of nested convex polygons?

2. How many 2-transmitters are always sufficient (and sometimes necessary) to cover the plane in the presence of a set of nested convex polygons?

Henceforth, we use the bounding box of a polygon to refer to the smallest axisparallel rectangle containing the polygon.

Some notation. We call a set of $k$ convex polygons $\left\{P_{1}, P_{2}, \ldots, P_{k}\right\}$ nested if $P_{1} \supseteq P_{2} \supseteq \cdots \supseteq P_{k}$. The total number of vertices of the set of polygons $\left\{P_{1}, P_{2}, \ldots, P_{k}\right\}$ is $n$.

Given such a set, we use the term layers for the boundaries of the polygons and rings for the portions of the plane between layers, i.e., the the $i$-th ring is $R_{i}=P_{i}-P_{i+1}$, for $i=1, \ldots, k-1$. In addition, $R_{0}=\mathbb{R}-P_{1}$ and $R_{k}=P_{k}$.

We assume that vertices on each layer have labels with indices increasing counterclockwise. Given a vertex $v_{j} \in P_{i}$, we call the positive angle $\angle v_{j-1} v_{j} v_{j+1}$ its external visibility angle. (Positive angles are measured counterclockwise, and negative angles are measured clockwise.) Its internal visibility angle is the negative angle $\angle v_{j-1} v_{j} v_{j+1}$.

Lemma 5. Placing a 2-transmitter at every other vertex in a given layer $i$ guarantees to completely cover layers $i-3, i-2, i-1$ and $i$, as well as rings $i-3$, $i-2$ and $i-1$.

Proof. The fact that layer $i$ is covered is obvious. As for the previous layers, notice that the convexity of $P_{i}$ guarantees that the external visibility angles of any vertex pair $v_{j}$ and $v_{j+2}$ overlap, as illustrated in Figure 4(a). Since $v_{j} \in$ $P_{i} \subseteq P_{i-1} \subseteq P_{i-2} \subseteq P_{i-3}$ and the polygons are convex, all rays from $v_{j}$ within its external visibility angle traverse exactly two segments before reaching layer $i-3$.

Lemma 6. Placing a 2-transmitter at each vertex of a given layer $i$ guarantees to completely cover layers $i-3, i-2, i-1, i, i+1, i+2$ and $i+3$, as well as rings $i-3, i-2, i-1, i, i+1$ and $i+2$.

Proof. The fact that layers $i-3, i-2, i-1, i$ and rings $i-3, i-2$ and $i-1$ are covered is a consequence of Lemma 5 . As for the remaining layers and rings, notice that, in the internal visibility angle of a 2 -transmitter $v_{j} \in P_{i}$, visibility is determined by the supporting lines from $v_{j}$ to layers $i+1, i+2$ and $i+3$, as illustrated in Figure 4(b). Having a 2-transmitter on each of the vertices of layer $i$, combined with the fact that all polygons are convex, guarantees total covering of layers $i+1, i+2$ and $i+3$ and rings $i, i+1$ and $i+2$. 


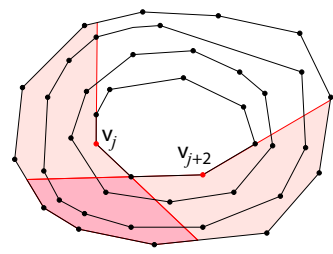

(a)

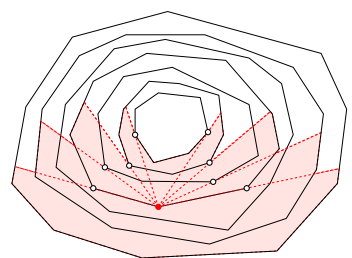

(b)

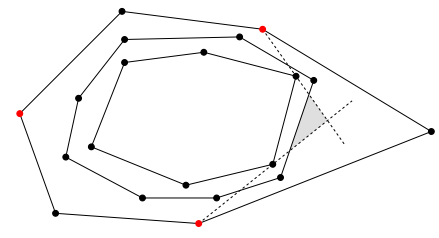

(c)

Fig. 4. (a) External visibility angles of two vertices $v_{j}, v_{j+2}$ of layer $i$. Only layers $i-3$, $i-2, i-1$ and $i$ are shown. (b) External and internal visibility from a 2-transmitter located in a vertex of layer $i$. Only layers $i-3, i-2, i-1, i, i+1, i+2$ and $i+3$ are shown. (c) The shaded region is not covered by the 2-transmitters located at the red vertices. Only the three involved layers are shown.

Theorem 4. $\left\lfloor\frac{n}{7}\right\rfloor+5$ 2-transmitters are always sufficient to cover the edges of any nested set of convex polygons with a total of $n$ vertices.

Proof. If the number of layers is $k \in\{1,2,3,4,5,6\}$, five 2-transmitters trivially suffice: one in the interior of $P_{k}$ and the other four at the corners of the bounding box of $P_{1}$. If $k \geq 7$, from the pigeonhole principle one of $i \in\{1,2,3,4,5,6,7\}$ is such that the set $G=\left\{P_{j} \mid j \in\{1, \ldots, k\}, j \equiv i(\bmod 7)\right\}$ has no more than $\left\lfloor\frac{n}{7}\right\rfloor$ vertices. Place one 2 -transmitter at each vertex of each $P_{j} \in G$. From Lemma 6 , for a certain value of $m \in \mathbb{Z}$ all edges in the following layers are covered: $i-3, i-2, i-1$ (if they exist), $i, \ldots, i+7 m, i+7 m+1, i+7 m+2$ and $i+7 m+3$ (if they exist). In the worst case, the only layers that may remain uncovered are 1,2 and 3 , as well as $k-2, k-1$ and $k$. Because of the convexity of the polygons, four 2-transmitters conveniently located at the corners of the bounding box of $P_{1}$, and one 2-transmitter located in the interior of $P_{k}$, can take care of covering these remaining layers. The total number of 2-transmitters used is at most $\left\lfloor\frac{n}{7}\right\rfloor+5$.

The transmitter placement from Theorem 4 guarantees that all edges are covered, while some rings remain uncovered.

Theorem 5. $\left\lfloor\frac{n}{6}\right\rfloor+3$ 2-transmitters are always sufficient to cover the plane in the presence of any nested set of convex polygons with a total of $n$ vertices.

Proof. The proof is similar to Theorem 4, but locating the 2-transmitters at all vertices of every $6^{\text {th }}$ layer (as opposed to every $7^{\text {th }}$ layer in Theorem 4 ).

Lemma 7. $\left\lfloor\frac{n}{8}\right\rfloor$ 2-transmitters are sometimes necessary to cover the plane in the presence of any nested set of convex polygons with a total of $n$ vertices.

Proof. This lower bound is established by the example from Figure 5, which shows four nested regular $t$-gons, with $t$ even (so $n=4 t$ ). Consider the set $S$ of midpoints of alternating edges of the outermost convex layer (marked $u_{i}$ 


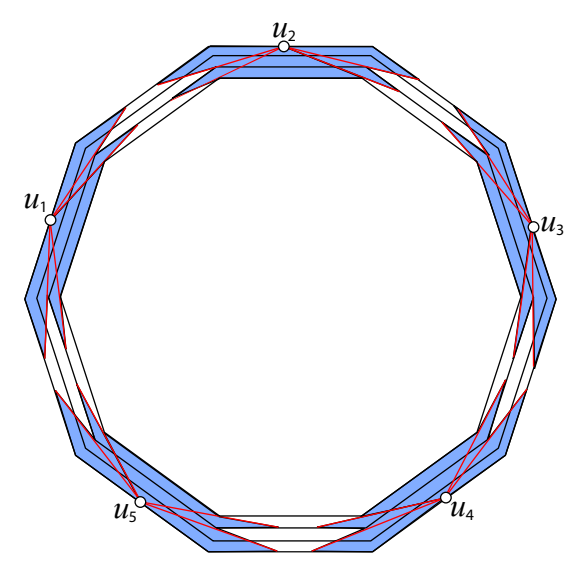

(a)

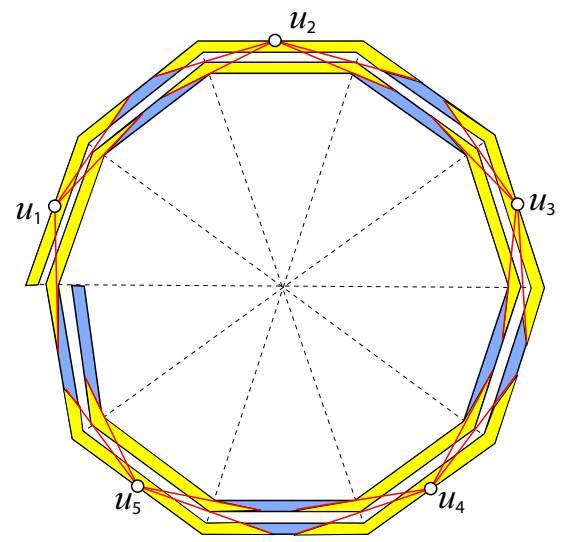

(b)

Fig. 5. (a) $\left\lfloor\frac{n}{8}\right\rfloor$ 2-transmitters are necessary to cover the edges of these four nested convex layers. (b) $\left\lfloor\frac{n}{8}\right\rfloor$ 2-transmitters are necessary to cover the edges of this spirangle polygon.

in Figure 5). The gap between adjacent layers controls the size of the visibility regions of the points in $S$ (by symmetry, all visibility regions have identical size). A small enough gap guarantees that the visibility regions of the points in $S$ are all disjoint, as illustrated in Figure 5. This means that at least $t / 2$ 2-transmitters are necessary to cover all points in $S$ (one transmitter in the visibility region of each point). So the number of 2 -transmitters necessary to cover all edges is $t / 2=n / 8$.

Lemmas 8 and 9 establish improved upper bounds for the case when all layers (convex polygons) have an even number of vertices. Due to space constraints, we omit the proofs of these lemmas.

Lemma 8. $\lfloor n / 8\rfloor+1$ 2-transmitters are always sufficient to cover the edges of any nested set of convex polygons with a total of $n$ vertices, if each of the polygons has an even number of vertices.

Lemma 9. $\left\lfloor\frac{n}{6}\right\rfloor+1$ 2-transmitters are always sufficient to cover the plane in the presence of any nested set of convex polygons with a total of $n$ vertices, if each of the polygons has an even number of vertices.

\section{Coverage of Simple Polygons}

This section addresses the problem of covering a polygonal region $P$ with 2transmitters placed interior to $P$. Therefore, when we talk about a vertex or an edge transmitter, the implicit assumption is that the transmitter is placed just inside the polygonal region, and so must penetrate one wall to reach the exterior. 
Our construction places a small (constant) number of transmitters outside $P$, but still within the bounding box for $P$.

\subsection{Lower Bounds For Covering Polygons}
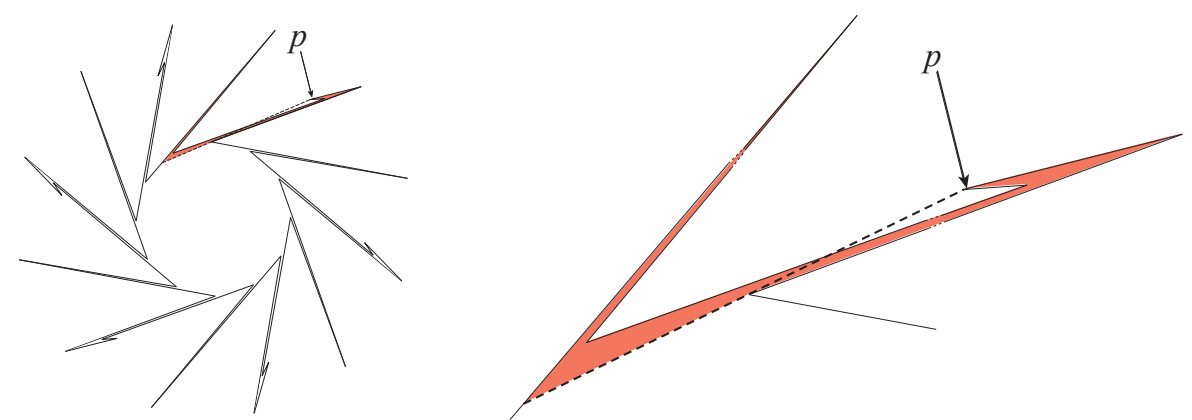

Fig. 6. A family of polygons requiring at least $n / 6$ interior 2 -transmitters to cover. For labeled point $p$ located in the tip of a barb (shown magnified on the right with the arms shortened), the locus of all interior points from which a 2-transmitter can cover $p$ is shown shaded.

Theorem 6. There are simple polygons that require at least $\frac{n}{6}$ 2-transmitters to cover when transmitters are restricted to the interior of the polygon.

Proof. Figure 6 shows the construction for a $n=36$ vertex polygon, which generalizes to $n=6 m$, for any $m \geq 2$. It is a pinwheel whose $n / 3$ arms alternate between spikes and barbs. Consider an interior point $p$ at the tip of a barb. The locus of all interior points from which a 2-transmitter can cover $p$ includes the spike counter-clockwise from the barb, the barb containing $p$, and a small section of the pinwheel center. This region is shown shaded for the point $p$ labeled in Figure 6. Observe that this shaded region is disjoint from the analogous regions associated with the other barb tips. Hence no two barb tips can be covered by the same 2-transmitter. Since there are $n / 6$ barbs, the lower bound is obtained.

\section{$3.2 \quad$ Spirangles}

Two edges are homothetic if one edge is a scaled and translated image of the other. A $t$-spirangle is a polygonal chain $A=a_{1}, a_{2}, \ldots, a_{m}$ that spirals inward about a center point such that every $t$ edges it completes a $2 \pi$ turn, and each edge pair $a_{i} a_{i+1}, a_{i+t} a_{i+1+t}$ is homethetic, for $1 \leq i \leq m-t$. We assume that the spiral direction is clockwise. A $t$-sided convex polygon may be thought of as 
generating a family of $t$-spirangles where the $i^{\text {th }}$ edge of each spirangle is parallel to the $(i \bmod t)^{t h}$ edge of the polygon, for $i=0,1,2, \ldots$. See Figure $7(\mathrm{a})$ for a 4 -spirangle example and a polygon generating it.

A homothetic t-spirangle polygon $P$ is a simple polygon whose boundary consists of two nested $t$-spirangles $A=a_{1}, a_{2}, \ldots, a_{m}$ and $B=b_{1}, b_{2}, \ldots, b_{m}$ generated by the same $t$-sided convex polygon, plus two additional edges $a_{1} b_{1}$ and $a_{m} b_{m}$ joining their endpoints. We assume that chain $B$ is nested inside of chain $A$, as shown in Figure $7(\mathrm{~b})$. We refer to $A$ as the convex chain and $B$ as the reflex chain in reference to the type of vertices found on each.

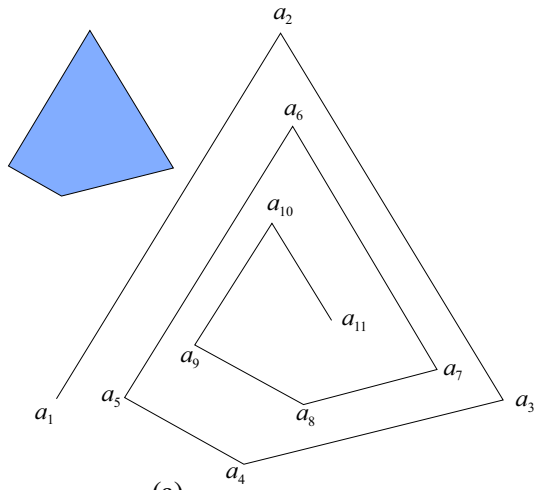

(a)

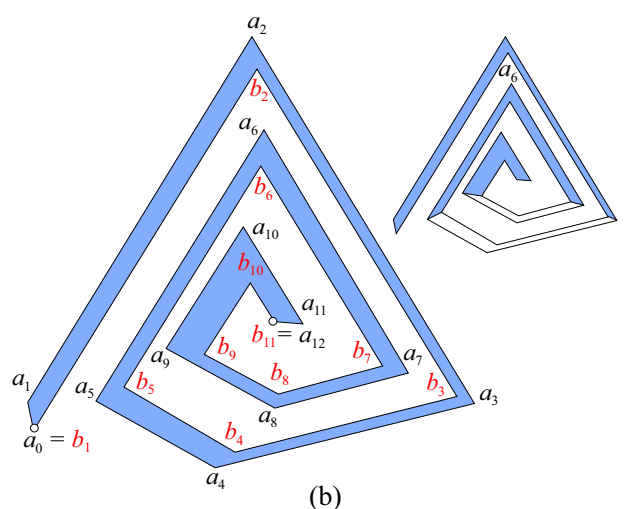

(b)

Fig. 7. Definitions (a) A 4-spirangle and corresponding convex polygon (b) Edgehomothetic spiral polygon (left) and quadrilaterals entirely visible to $a_{6}$ (right).

Property 1. Let $P$ be a homothetic spirangle polygon, composed of a convex spirangle $A=a_{1}, a_{2}, \ldots$, and a reflex spirangle $B=b_{1}, b_{2}, \ldots$. Then $a_{i}$ and $b_{i}$ see each other, and the set of diagonals $\left\{a_{i} b_{i} \mid i=1,2, \ldots\right\}$, induces a partition of $P$ into quadrilaterals. Furthermore, the visibility region of $a_{i}$ includes six quadrilaterals: two quadrilaterals adjacent to $a_{i-t} b_{i-t}$, two adjacent to $a_{i} b_{i}$, and two adjacent to $a_{i+t} b_{i+t}$. See right of Figure 7(b).

Theorem 7. $\left\lfloor\frac{n}{8}\right\rfloor$ 2-transmitters are sufficient, and sometimes necessary, to cover a homothetic t-spirangle polygon $P$ with $n$ vertices.

Proof. The algorithm that places transmitters at vertices of $P$ to cover the interior of $P$ is fairly simple, and is outlined in Table 1.

The proof that this algorithm covers the interior of $P$ is fairly intuitive. Due to space constraints, we omit this proof. The fact that the $\left\lfloor\frac{n}{8}\right\rfloor$ bound is tight is established by the spirangle polygon example from Figure 5(b), and the arguments are similar to the one used in the proof of Lemma 7. The example from Figure 5(b) depicts a worst-case scenario, in which transmitters do not get the chance to use their full coverage potential, since the total turn angle of the spirangle is between $2 \pi$ and $6 \pi$. 


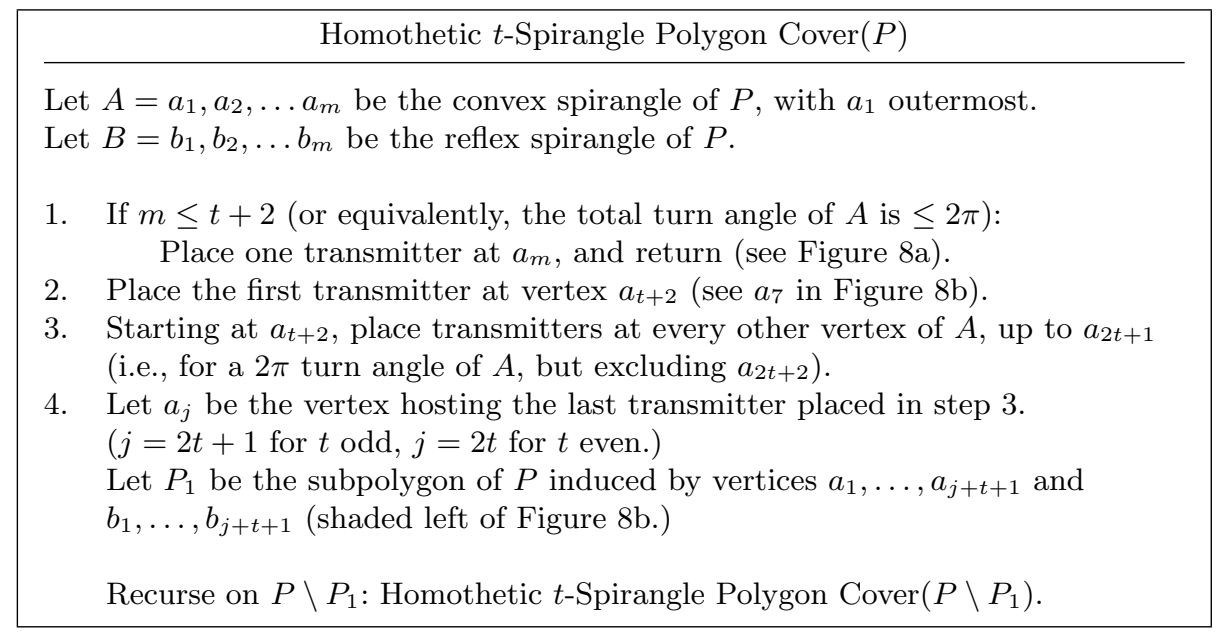

Table 1. Covering the interior of a homothetic spirangle polygon with 2-transmitters.

\subsection{Arbitrary Spirals}

A spiral polygon $P$ consists of a clockwise convex chain and a clockwise reflex chain that meet at their endpoints. A trivial $\left\lfloor\frac{n}{4}\right\rfloor$ upper bound for the number of 2-transmitters that are sufficient to cover $P$ can be obtained as follows. Pick the chain $\Gamma$ of $P$ with fewer vertices (i.e., $\Gamma$ is the reflex chain of $P$, if the number of reflex vertices exceeds the number of convex vertices, and the convex chain of $P$ otherwise). Then simply place one vertex 2 -transmitter at every other vertex of $\Gamma$. By definition, the visibility ray from one 2-transmitter can cross the boundary of $P$ at most twice. Note however that, even under the restriction that transmitters be placed interior of $P$, the visibility ray of one transmitter can leave and re-enter $P$, as depicted in Fig. 9(a) for transmitter labeled $a$. Then arguments similar to the ones used in Lemma 5 show that the union of the external visibility angles of all these 2-transmitters cover the entire plane. So we have the following result:

Lemma 10. $\left\lfloor\frac{n}{4}\right\rfloor$ 2-transmitters placed interior to an arbitrary polygonal spiral $P$ are sufficient to cover $P$ (in fact, the entire plane).

We remark on two special situations. In the case of transmitters placed at every other reflex vertex of $P, 0$-transmitters are sufficient to cover the interior of $P$, and 1-transmitters are sufficient to cover the entire plane. In the case of transmitters placed at every other convex vertex of $P, 1$-transmitters are sufficient to cover $P$, if they are placed outside of $P$.

An improved upper bound can be established for non-degenerate spirals, which we define as spirals in which each $2 \pi$-turn of each of the convex and reflex chain of $P$ is homothetic to a convex polygon (i.e., it contains at least 3 vertices). The result (whose proof we omit due to space restrictions) is as follows. 


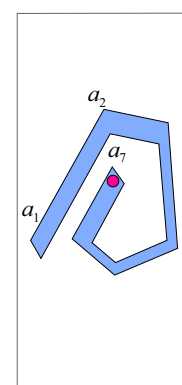

(a)

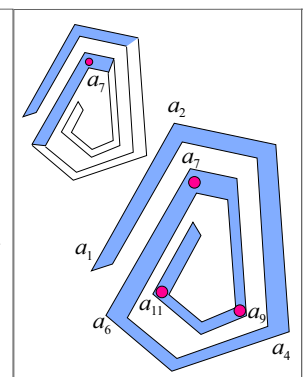

(b)

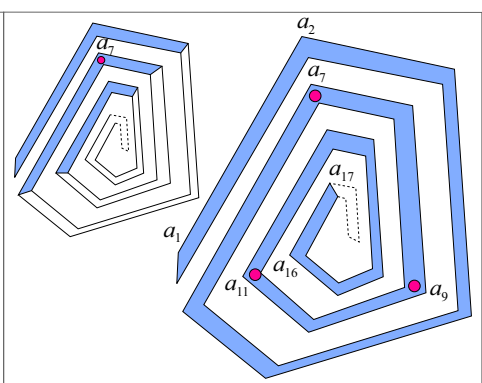

(c)

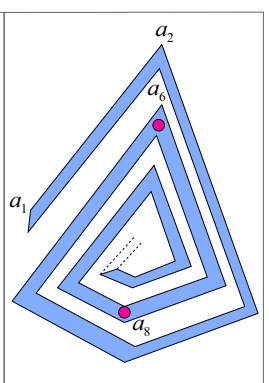

(d)

Fig. 8. Covering spirangles with 2-transmitters. (a) A $t$-spirangle $(t=5)$ with $2 t+4$ edges covered with one transmitter. (b) A $t$-spirangle $(t=5)$ with $8 t$ edges. (c) A $t$ spirangle $(t=5)$ with $6 t+4$ edges covered with $t / 2+1$ transmitters. (d) A $t$-spirangle $(t=4)$ with $6 t$ edges covered with $t / 2$ transmitters.

Lemma 11. Let $P$ be a polygonal spiral whose every $2 \pi$ turn chain has at least 3 vertices. Then $\left\lceil\frac{2 n}{9}\right\rceil+1$ 2-transmitters placed interior to $P$ are sufficient to cover the interior of $P$ (in fact, the entire plane).

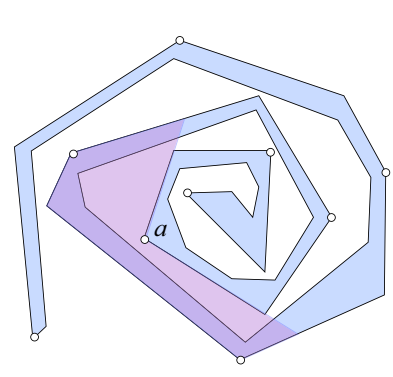

(a)

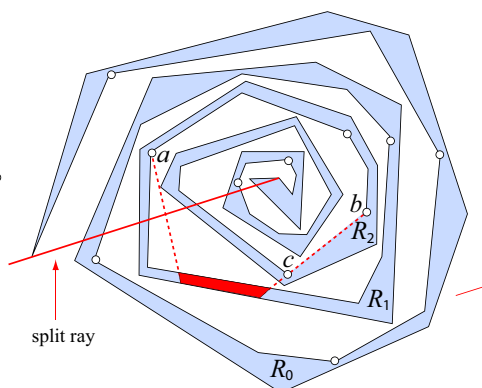

(b)

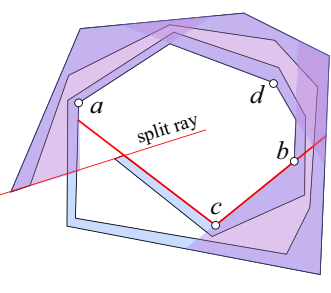

(c)

Fig. 9. Transmitters marked with small circles (a) Visibility angle of $a$ (b) The dark area is not covered by $a$ or $b$ (c) $P$ is covered.

\section{Conclusion}

In this paper we study the problem of covering ("guarding") a target region in the plane with $k$-transmitters, in the presence of obstacles. We develop lower and upper bounds for the problem instance in which the target region is the plane, and the obstacles are lines and line segments, a guillotine subdivision, or nested convex polygons. We also develop lower and upper bounds for the problem 
instance in which the target region is the set of rings created by nested convex polygons, or the interior of a spiral polygon. Our work leaves open two main problems: (i) closing the gap between the $\left\lfloor\frac{n}{8}\right\rfloor$ lower bound and the $\left\lfloor\frac{n}{6}\right\rfloor$ upper bound in the case of nested convex layers, and (ii) closing the gap between the $\left\lfloor\frac{n}{8}\right\rfloor$ lower bound and the $\left\lfloor\frac{n}{4}\right\rfloor$ upper bound for spiral polygons. Investigating the $k$-transmitter problem for other classes of polygons (such as orthogonal polygons) also remains open.

Acknowledgement. We thank Joseph O'Rourke for the pinwheel example from Fig. 6 and for initiating this line of work.

\section{References}

1. O. Aichholzer, F. Aurenhammer, F. Hurtado, P. Ramos, and J. Urrutia. $k$-convex polygons. In Euro CG, pages 117-120, 2009.

2. O. Aichholzer, R. Fabila-Monroy, D. Flores-Pealoza, T. Hackl, C. Huemer, J. Urrutia, and B. Vogtenhuber. Modem illumination of monotone polygons. In EuroCG, 2009 .

3. O. Borodin. A new proof of the 6 color theorem. Journal of Graph Theory, 19(4):507-521, 1995.

4. T. Christ, M. Hoffmann, Y. Okamoto, and T. Uno. Improved bounds for wireless localization. In SWAT '08: Proceedings of the 11th Scandinavian workshop on Algorithm Theory, pages 77-89, Berlin, Heidelberg, 2008. Springer-Verlag.

5. V. Chvátal. A combinatorial theorem in plane geometry. Journal of Combinatorial Theory Series B, 18:39-41, 1975.

6. J. Czyzowicz, E. Rivera-Campo, N. Santoro, J. Urrutia, and J. Zaks. Guarding rectangular art galleries. Discrete Applied Math, 50:149-157, 1994.

7. M. Damian, R. Flatland, J. O'Rourke, and S. Ramaswami. A new lower bound on guard placement for wireless localization. In FWCG 07: Proc. of the 17th Fall Workshop on Computational Geometry, pages 21-24, November 2007.

8. A. M. Dean, W. Evans, E. Gethner, J. Laison, M. A. Safari, and W. T. Trotter. Bar $k$-visibility graphs: Bounds on the number of edges, chromatic number, and thickness. In Proc. of Graph Drawing, LNCS 3843, page 7382, 2005.

9. D. Eppstein, M. T. Goodrich, and N. Sitchinava. Guard placement for efficient point-in-polygon proofs. In SoCG, pages 27-36, 2007.

10. R. Fabila-Monroy, A. R. Vargas, and J. Urrutia. On modem illumination problems. In XIII Encuentros de Geometria Computacional, Zaragoza, Spain, June 2009.

11. S. Felsner and M. Massow. Parameters of bar $k$-visibility graphs. Journal of Graph Algorithms and Applications, 12(1):5-27, 2008.

12. R. Fulek, A. F. Holmsen, and J. Pach. Intersecting convex sets by rays. Discrete Comput. Geom., 42(3):343-358, 2009.

13. S. G. Hartke, J. Vandenbussche, and P. Wenger. Further results on bar $k$-visibility graphs. SIAM Journal of Discrete Mathematics, 21(2):523-531, 2007.

14. D. T. Lee and A. K. Lin. Computational complexity of art gallery problems. IEEE Trans. Inf. Theor., 32(2):276-282, 1986.

15. J. O'Rourke. Art gallery theorems and algorithms. Oxford University Press, Inc., New York, NY, USA, 1987.

16. J. Urrutia. Art gallery and illumination problems. In J.-R. Sack and J. Urrutia, editors, Handbook of Computational Geometry, pages 973-1027. North-Holland, 2000 . 